\title{
Chick embryo chorioallantoic membrane model systems to study and visualize human tumor cell metastasis
}

\author{
Elena I. Deryugina $\cdot$ James P. Quigley
}

Accepted: 23 October 2008 / Published online: 13 November 2008

(C) Springer-Verlag 2008

\begin{abstract}
Since their introduction almost a century ago, chick embryo model systems involving the technique of chorioallantoic grafting have proved invaluable in the in vivo studies of tumor development and angiogenesis and tumor cell dissemination. The ability of the chick embryo's chorioallantoic membrane (CAM) to efficiently support the growth of inoculated xenogenic tumor cells greatly facilitates analysis of human tumor cell metastasis. During spontaneous metastasis, the highly vascularized CAM sustains rapid tumor formation within several days following cell grafting. The dense capillary network of the CAM also serves as a repository of aggressive tumor cells that escaped from the primary tumor and intravasated into the host vasculature. This spontaneous metastasis setting provides a unique experimental model to study in vivo the intravasation step of the metastatic cascade. During experimental metastasis when tumor cells are inoculated intravenously, the CAM capillary system serves as a place for initial arrest and then, for tumor cell extravasation and colonization. The tissue composition and accessibility of the CAM for experimental interventions makes chick embryo CAM systems attractive models to follow the fate and visualize microscopically the behavior of grafted tumor cells in both spontaneous and experimental metastasis settings.
\end{abstract}

Keywords Chick embryo CAM models .

Tumor cell metastasis · Intravasation · Angiogenesis · Live cell imaging

E. I. Deryugina $(\bowtie) \cdot$ J. P. Quigley

The Scripps Research Institute, 10550 North Torrey Pines Road, La Jolla, CA 92037, USA

e-mail: deryugin@scripps.edu

\section{Introduction}

Complementing murine models for tumor cells dissemination, chick embryo model systems offer a number of unique advantages to study the complex, multistep process of tumor cell metastasis. Since the lymphoid system is not fully developed till late stages of incubation, the chick embryo serves as a naturally immunodeficient host capable of sustaining grafted tissues and cells without species-specific restrictions. Different chick embryo model systems allow for comprehensive analysis of specific stages and aspects of cancer cell dissemination such as tumor cell intravasation in the spontaneous metastasis model, tumor cell colonization in the experimental metastasis model or tumor-induced angiogenesis in the collagen onplant model. The core of these model systems is the use of a specialized tissue, i.e., chorioallantoic membrane (CAM), which provides a uniquely supportive environment for primary tumor formation and a source of angiogenic blood vessels. In addition to nurturing developing xenografts, the CAM blood vessel network provides conduits for tumor cell intravasation, dissemination, and vascular arrest and finally, a repository where arrested cells extravasate to form micro metastatic foci. Due to the ease of repetitive experimental manipulations before or after tumor cell grafting, the CAM models allow one to identify individual steps of the metastatic cascade where specific molecules or biochemical processes manifest their functional involvement. This review will emphasize the experimental approaches based on CAM models and also highlight histological analyses of human tumor cells, which have been successfully employed in our laboratory to mechanistically address the functionality of several metastasisrelated molecules. 
CAM development, structure, and imaging

During chick embryo incubation, the CAM is formed between days 5 and 6 by partial fusion of chorion and allantois (Melkonian et al. 2002; Romanoff 1960). The CAM, functionally serving as lungs of the embryo, develops fast and surrounds the whole embryo by day 12 of incubation. Histologically, the CAM contains three major layers, i.e., the ectoderm attached to the shell membrane, the mesoderm enriched in blood vessels and stromal components, and the endoderm facing the allantoic cavity. By day 10 of incubation, the CAM also comprises the fully developed ectoderm capillary plexus, which represents a network of tiny capillaries connecting the arterial and venous blood vessel networks.

The CAM is a very thin structure, rarely exceeding $100 \mu \mathrm{m}$ across the entire three layers. Hematoxylin-eosin staining of paraffin sections clearly identifies the ectoderm, represented by a one- or two-cell epithelial layer; the capillary plexus visualized as tiny circular openings in the ectoderm frequently filled with erythrocytes; the mesoderm filled with the stromal cells, collagen fibers and blood vessels of different diameters, including terminal capillaries localized right under the ectoderm; and the one cell-layer of flat endoderm (Fig. 1a).

If the chick embryo is injected with fluorescent-tagged lectin such as Lens culinaris agglutinin (LCA), the intact vascular network of the CAM can be visualized by a top planar view of whole-mount preparations in a fluorescent microscope (Fig. 1b). Complementing conventional histology, the immunofluorescence evaluation provides a more discernable radiation of terminal arterial and venous capillaries forming a dense capillary network of the ectodermal
Fig. 1 Ectoderm capillary plexus of day 12 chick embryo. a H\&E staining. b Immunofluorescent staining with Lens culinaris agglutinin (LCA). c, $\mathbf{d}$ Immunohistochemical staining with endothelium-specific lectin Sambuco negro agglutinin (SNA). d DIC microscopy of live, non-fixed whole mounts of the CAM at the ectoderm plexus level (e) and mesoderm level (f), $\times 100(\mathbf{a}, \mathbf{c})$ and $\times 200(\mathbf{b}, \mathbf{d}-\mathbf{f})$
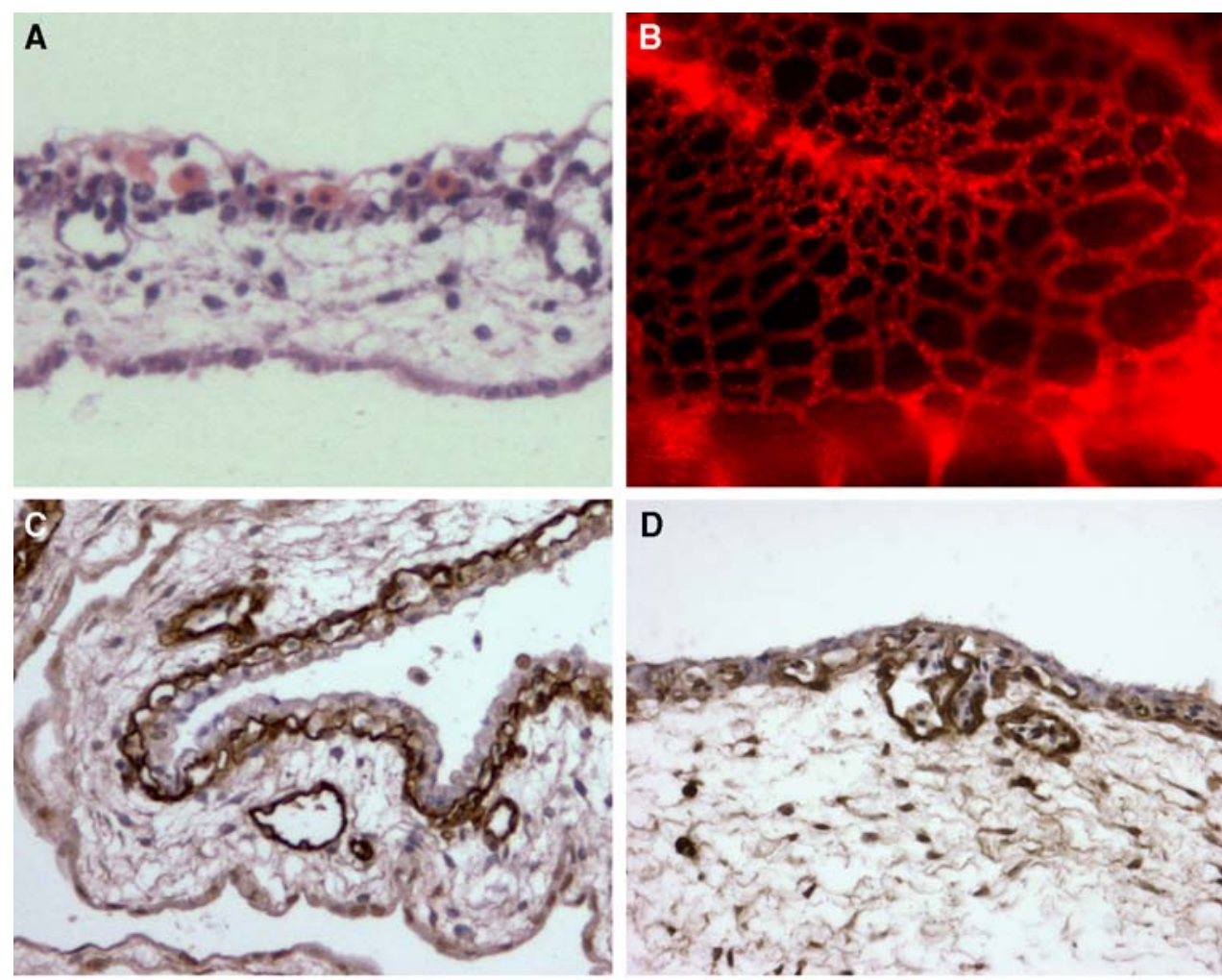

D
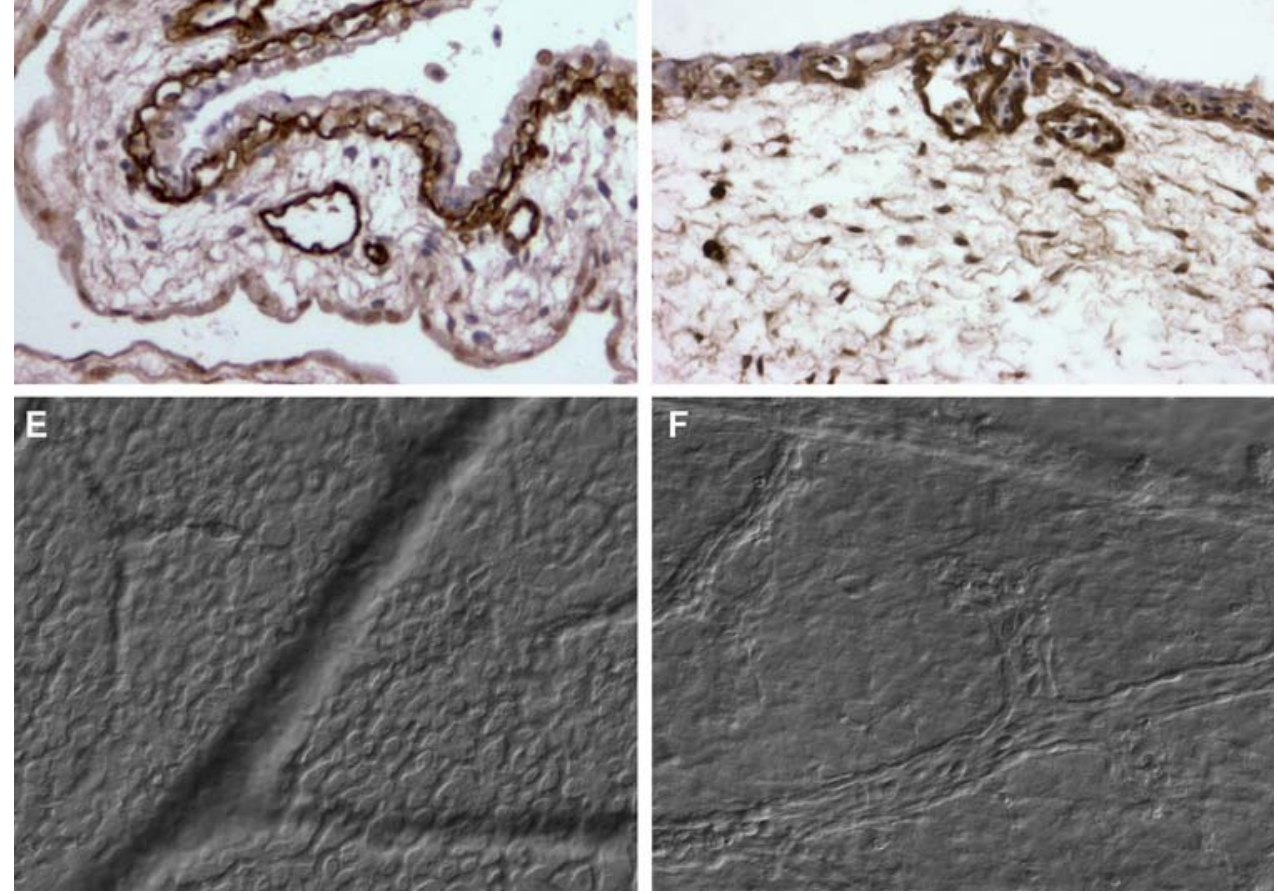
plexus. This plexus comprises capillaries of so small diameter (approximately that of one erythrocyte) and such high density that the whole vascular network generates a honey comb structure, allowing for maximal surface and, therefore maximal levels of gas and nutrient exchanges.

Highlighting the vasculature with Sambuco negro agglutinin (SNA), which specifically binds to chicken endothelium, results in a better discrimination of the ectoderm capillary plexus and blood vessels within CAM mesoderm (Fig. 1c). Analysis under higher magnification (Fig. 1d) demonstrates tiny capillaries stained brown with SNA and appearing embedded into the thin layer of ectoderm cells counterstained blue with hematoxylin. Structural organization of the CAM can be also appreciated by DIC microscopy of the CAM stretched out on the glass slides. Since the CAM is so thin, differential focusing allows for detailed visualization of different planes: the ectoderm surface, the capillary plexus (Fig. 1e), the vasculature network (Fig. 1f), and the endoderm.

The ectoderm capillary plexus is one of the most important histological features of the CAM pertinent to chick embryo metastasis and angiogenesis models since it efficiently supports tissue grafting via rapid neovascularization and also serves as a repository of experimentally inoculated or spontaneously intravasated tumor cells.

\section{CAM model for spontaneous tumor cell intravasation and metastasis}

The highly vascularized nature of the CAM greatly promotes the efficiency of tumor cell grafting (Armstrong et al. 1982; Murphy 1913; Ossowski and Reich 1980). Tumor cells are grafted in ovo in 20-30 $\mu \mathrm{l}$ cell inoculums introduced through a small window made in the shell above the lowered (i.e., "dropped") CAM. The CAM "dropping" is performed by making an air pocket between separated shell membrane and the CAM. Depending on the tissue origin, number of tumor cells and their proliferation capacity, primary CAM tumors can reach up to $500-600 \mathrm{mg}$ in 67 days after cell inoculation. Remarkably, within 5-7 days not only do aggressive tumor cells develop sizable tumors, but they can escape the primary site, invade surrounding stroma, intravasate into blood vessels, and reach distal portions of the CAM and internal organs, where disseminated cells extravasate and form micro metastasis foci. Therefore, similar to the murine model, all steps of the multistep metastatic cascade are recapitulated in the chick embryo spontaneous metastasis model but, importantly, in a very short period of time.

Immunohistochemical detection makes it possible to localize human tumor cells within the chick embryo tissue background and therefore identify specific places where aggressive tumor cells manifest their differential ability to spontaneously metastasize. Such analysis was initially performed by employing the high and low disseminating variants of human HT-1080 fibrosarcoma (HT-hi/diss and HT-lo/diss, respectively), which were generated in our laboratory by in vivo selection for a 50-100-fold difference in their ability to intravasate and disseminate during spontaneous metastasis in the chick embryo (Deryugina et al. 2005). Importantly, these fibrosarcoma dissemination variants give rise to primary tumors of similar sizes, thus excluding inefficiency in the grafting or in vivo proliferation as the reasons responsible for the lack of intravasation in HT-lo/ diss cells.

The detailed immunohistological kinetic analysis of HT-hi/diss dissemination was performed by staining CAM tissue and liver with mAb 29-7, which was generated by subtractive immunization and demonstrated to specifically recognize human CD44. After $24 \mathrm{~h}$ following cell grafting, topically applied HT-hi/diss cells appear already localized under the CAM ectoderm. Two to three days later, they form a cellular mass with numerous mitosis structures among tumor cells forming a primary tumor. Interestingly, while HT-lo/diss tumors are characterized by a rather defined tumor-stroma border, the border of HT-hi/diss tumors is more distorted as HT-hi/diss cells appear to invade CAM stroma (Fig. 2a) and migrate towards, or wrap themselves around blood vessels (Fig. 2b). This type of tumor cell behavior was defined by us as "vasculotropism" since the cells appeared to be specifically attracted to blood vessels rather than distributed randomly within CAM mesoderm. Frequently, HT-hi/diss cells could be visualized as being integrated into a blood vessel wall. By day 3 after cell grafting, the first HT-hi/diss cells can be visualized intravascularly in the blood vessels adjacent to the primary tumor, thereby manifesting the beginning of intravasation (Deryugina et al. 2005).

The use of fluorescent-tagged tumor cells provides additional advantages allowing their visualization in live, nonfixed CAM with the vasculature differentially highlighted by fluorescence-tagged LCA. When GFP-labeled HT-1080 cells were used in the spontaneous metastasis assay, few HT-lo/diss cells were found escaping primary tumor sites and those that escaped appeared fragmented and randomly scattered among blood vessels (Fig. 2C1). Interestingly, primary HT-hi/diss tumors presented intra-tumoral vasculature that appeared distorted and dilated and sometimes encompassing intravascular tumor foci. In accordance with initial observations indicating vasculotropism of HT-hi/diss cells, tumor cells escaping the primary site were found along blood vessels proximal to the tumor border (Fig. 2C2). Quantification of tumor cells localized in a close proximity to blood vessels demonstrated a sixfold differential between HT-hi/diss over HT-lo/diss intravasation variants (Fig. 2d). 
Fig. 2 Vasculotropism of HThi/diss cells escaped from primary CAM tumors. HT-lo/diss cells (A1) and HT-hi/diss cells (A2) were placed onto the CAM of day 10 incubation chick embryo. Paraffin sections of day 5 primary tumors were stained with antiCD44 mAb 29-7 (brown) and counterstained with Mayer's hematoxylin, $\times 200$. HT-hi/diss cells in the vicinity of tumor/ stroma border appear to be integrated into the wall of blood vessel (b), $\times 400$. GFP-expressing HT-lo/diss cells (C1) and HT-hi/ diss cells (C2) escaping CAM primary tumors were visualized in the embryos with the vasculature highlighted with red fluorescent-tagged LCA, $\times 400$. d Quantitative analysis of HT-lo/ diss and HT-hi/diss cells associated with blood vessels
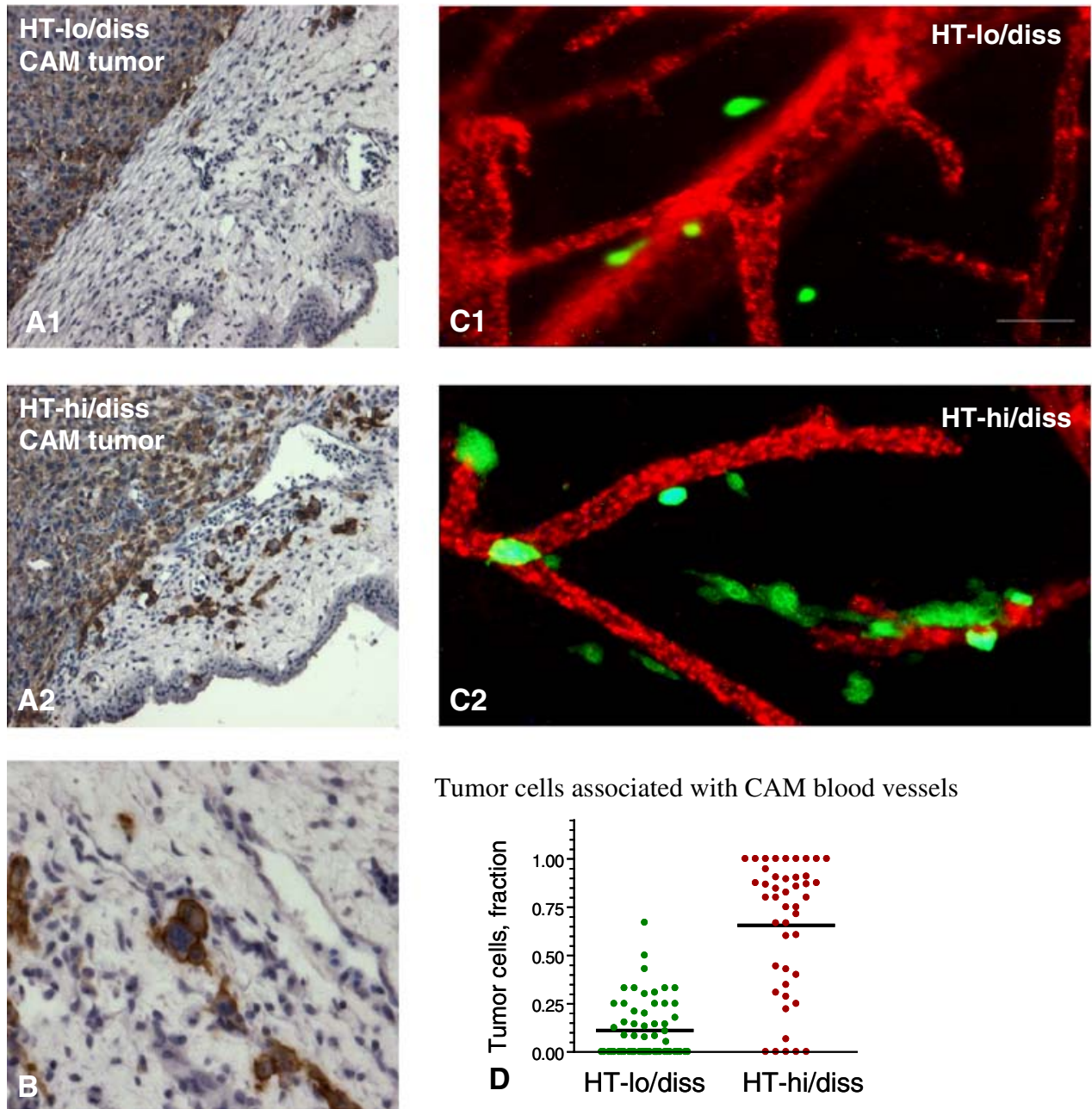

Tumor cells associated with CAM blood vessels

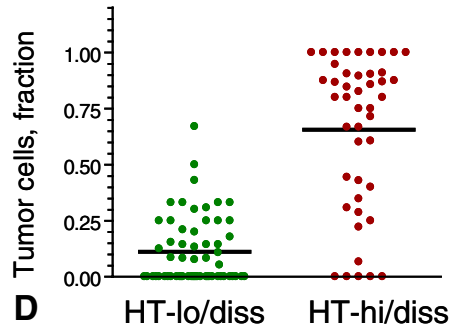

By day 4 after cell grafting, intravascular HT-hi/diss cells can be identified in the portions of CAM distal to the site of primary tumor development, confirming their vascular route of dissemination (Fig. 3a-d). It is interesting that almost all intravasated cells initially appeared trapped in the ectodermal plexus, most probably due to size restriction. Later, disseminating HT-hi/diss cells appear extravasating from terminal capillaries (Fig. 3e) and migrating within the CAM mesoderm (Fig. 3f). These extravasated cells are highly migratory since many were found in the middle portions of the CAM mesoderm. Disseminated HThi/diss cells are also highly proliferative, generating quite impressive metastatic foci by day 5-7 following grafting. Remarkably, very few GFP-labeled HT-lo/diss cells could be identified by immunofluorescence microscopy in distal portions of the CAM.

Actual levels of intravasation and vascular dissemination of human tumor cells in the chick embryo could be determined by a very sensitive quantitative PCR analysis based on amplification of Alu DNA repeats characteristic of the primate genome (Schmid and Jelinek 1982). This Alu qPCR analysis confirmed very low levels of
HT-lo/diss dissemination and indicated the onset of intravasation of HT-hi/diss cells between days 3 and 4 after tumor cell grafting and steadily increasing levels of intravasation occurring during the next 3-4 days (Deryugina et al. 2005).

In parallel to metastatic growth in the distal CAM, intravasated HT-hi/diss cells could be identified in the internal organs of the embryo such as liver and lungs by both Alu qPCR and immunohistochemistry. Initially, tumor cells in the liver display intravascular localization (Fig. 4a), but with time generate clear extravascular micro metastatic foci (Fig. 4b).

CAM models for analysis of tumor vasculotropism in vivo

In search for the mechanisms underlying a functional differential in spontaneous metastasis between HT-lo/diss and HT-hi/diss cell variants, we performed in vivo live imaging to analyze distinct migration patterns exhibited by HT-hi/ diss versus HT-lo/diss cells in vivo. In this assay, the mixtures of GFP-labeled and non-labeled cells (1:20) were injected directly into mesodermal CAM layer of the 
Fig. 3 Intravasated GFP-labeled HT-hi/diss cells visualized intravascularly (a-d) or appearing extravasating (e) and scattering (f) in the whole mount CAM preparations as visualized by DIC microscopy (a), DIC microscopy coupled with the highlighting of the CAM vasculature with the red-fluorescent LCA (b), and fluorescent microscopy $(\mathbf{c}-\mathbf{f}), \times 200$
Fig. 4 Immunohistochemical staining of HT-hi/diss cells spontaneously metastasized to the liver of chick embryos bearing CAM tumors. Left HT-hi/ diss cells localized intravascular, right extravascular micrometastatic foci, $\times 200$
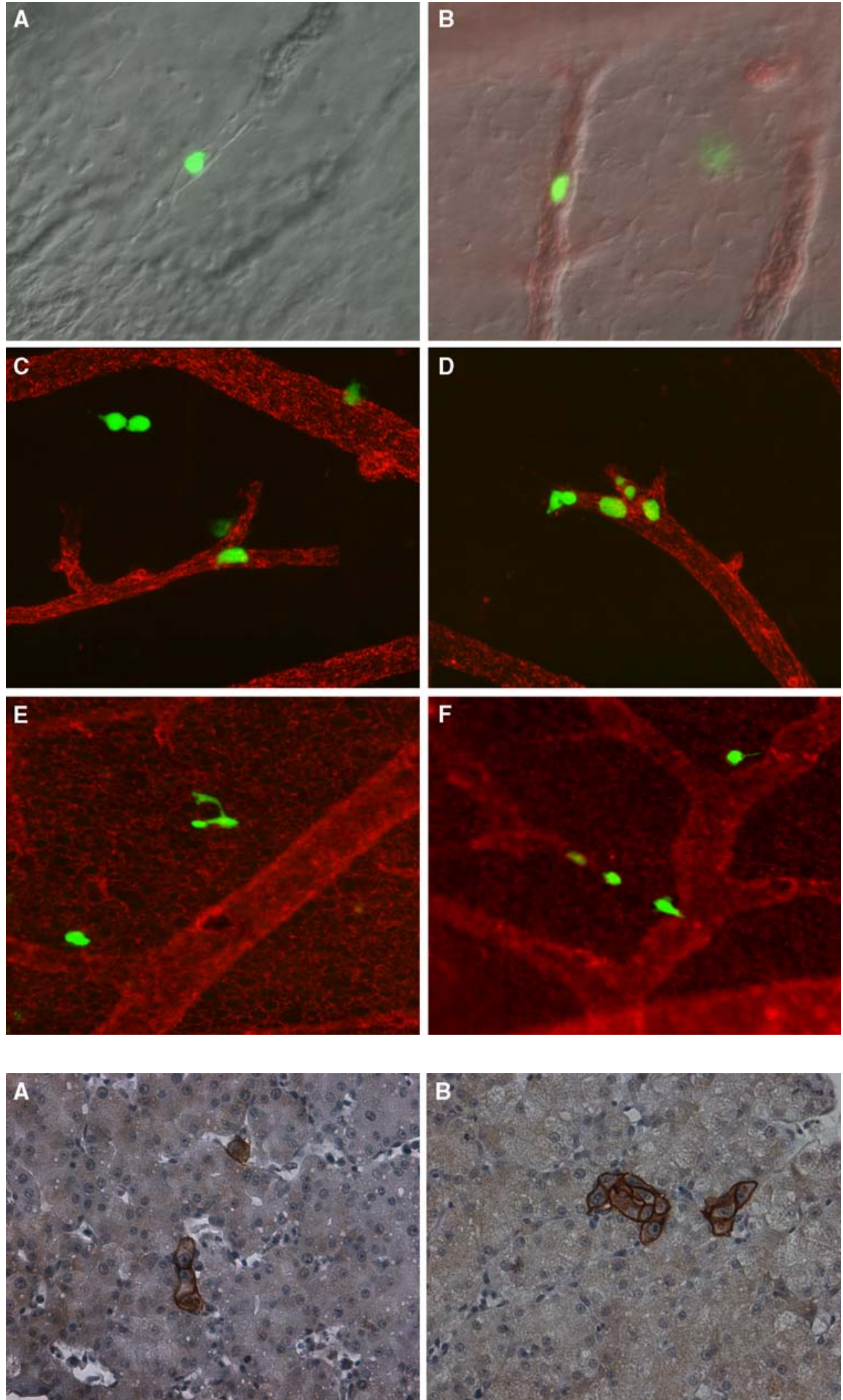

thermostat platform and a videocamera. A planar focus was chosen at the microtumor/CAM stroma border enriched in blood vessels. embryos grown ex ovo and allowed to form microtumors for 3 days (Fig. 5, left panels). The live embryos were then placed under the objective of a microscope equipped with a 
Fig. 5 Migration of HT-1o/diss (a) and HT-hi/diss (b) cells from the microtumors (left panels) developed in the CAM mesoderm. Intravital microscopy was performed in live embryos with an Olympus microscope equipped with a videocamera. Right panels depict enlarged portions of the frames originally taken with a $\times 20$ objective
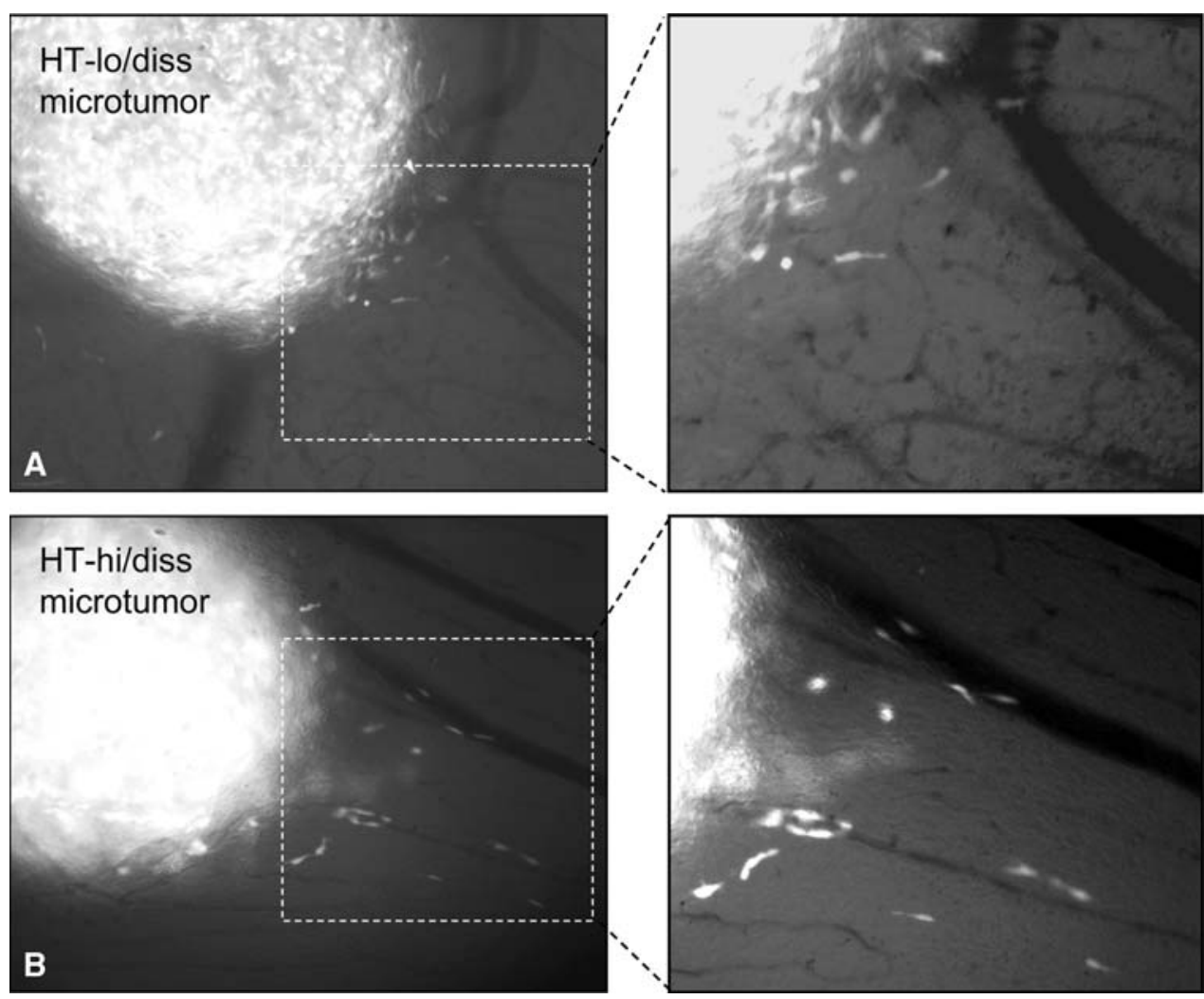

Fig. 6 Vasculotropism of HThi/diss cells escaped from microtumors developed on the CAM of the chick embryos grown ex ovo. a Shell-less chick embryo with HT-hi/diss microtumors (circles) developed for 5 days after grafting GFPlabeled cells within a drop of matrigel. b HT-hi/diss microtumor at larger magnification. c, d GFP-labeled HT-hi/diss cells visualized along CAM blood vessels by overlaying DIC and fluorescence digital images (c) or depicting overlaid immunofluorescent images only (c), $\times 200$

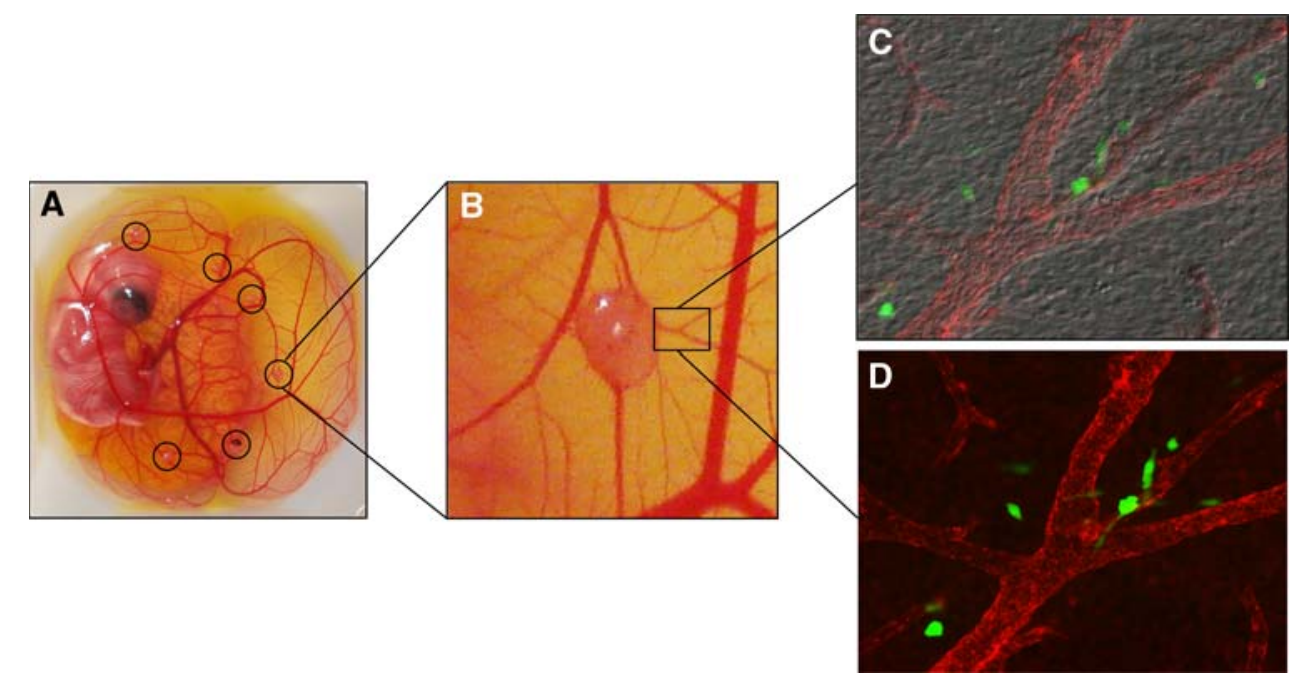

Twelve-hour video microscopy demonstrated that both HT-hi/diss and HT-lo/diss manifested high locomotion activity within tumors and at tumor-stroma border. However, while HT-lo/diss cells after leaving the microtumor appeared to be drawn back to the primary site, some of HT$\mathrm{hi} /$ diss were persistent in their directional migration away from the tumor (Fig. 5, right panels). Notably, this directional migration of HT-hi/diss cells was associated with locomotion towards or along CAM blood vessels, therefore manifesting cell behavior that has been defined by the above-introduced term "vasculotropism".
In an attempt to facilitate quantification of vasculotropic migration of tumor cells, we recently established an in vivo modification of the above-described model. If GFP-labeled HT-hi/diss cells are placed within a small volume of matrigel on the CAM of embryos incubated ex ovo (Fig. 6a), they form micro-tumors easily visualized after 3-4 days of incubation (Fig. 6b). GFP-expressing cells escaped from primary microtumors were found closely associated with blood vessels of the CAM observed with DIC-equipped fluorescent microscope (Fig. 6c, d). 
Fig. 7 Influx of inflammatory cells to CAM tumors developed for 6 days from HT-lo/diss and HT-hi/diss cells. Heterophils (A1 and A2) were visualized with specific anti chicken MMP9 antibody. Monocyte/macrophages (B1 and B2) were highlighted with anti-MMP-13 antibody, $\times 200$. Density of inflammatory cells was determined in digital images and presented in the corresponding graphs (A3 and B3)
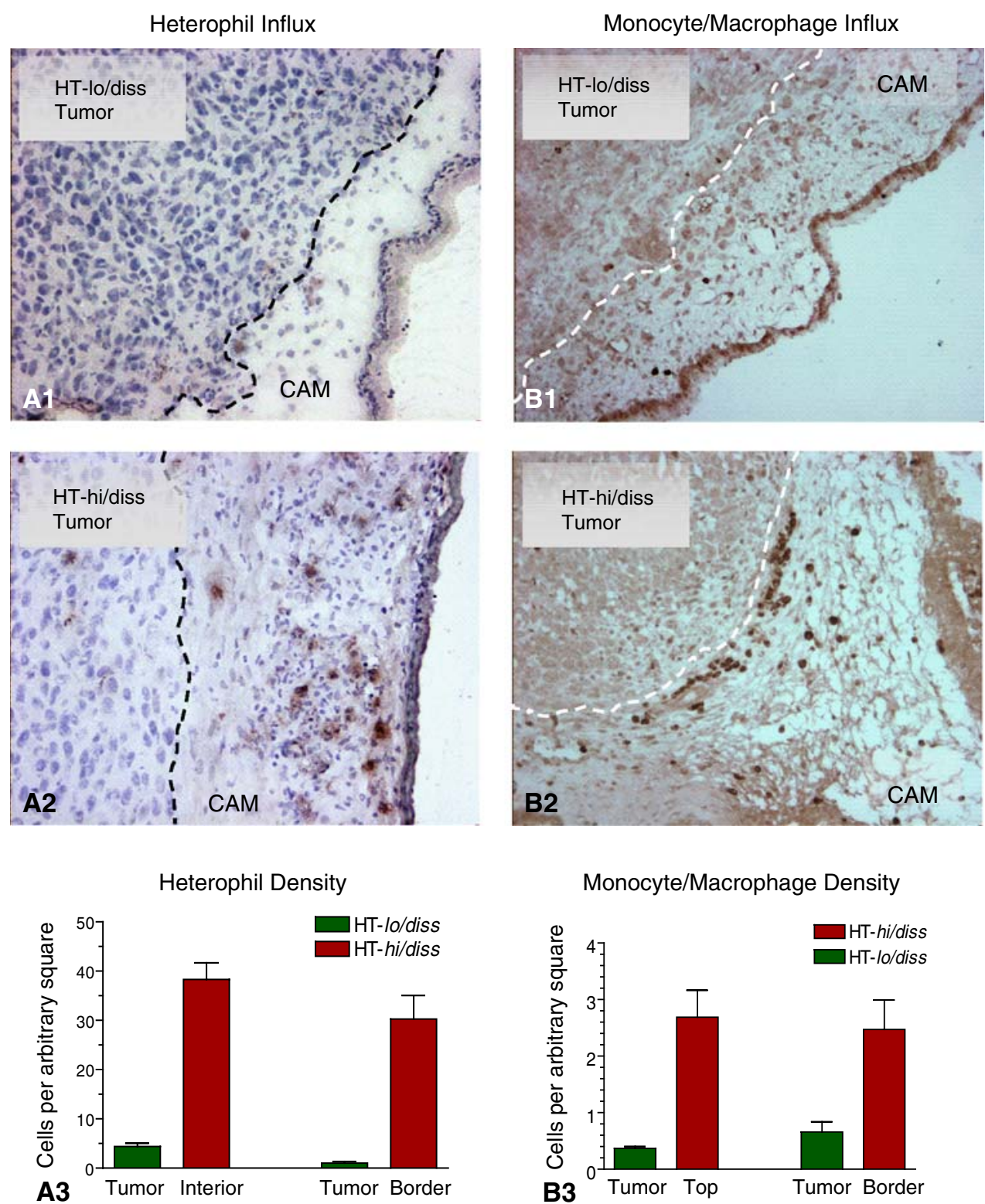

Inflammatory cell influx into CAM primary tumors

Inflammatory cells constitute an important cellular component of the primary tumor microenvironment, which paradoxically can perform anti-tumor surveillance in parallel with facilitating tumor progression (Balkwill et al. 2005; Condeelis and Pollard 2006; de Visser et al. 2006; van Kempen et al. 2006). To analyze whether in addition to the differential in intravasation capacity, our HT-1080 variants exhibited differential in the capacity to induce inflammatory cell influx, we performed immunohistochemical analysis of inflammatory cells infiltrating primary CAM tumors.

The two major inflammatory cell types present in day 10-15 embryos are heterophils and monocytes. Heterophils functionally serve as an avian analogue of mammalian neutrophils and represent a main source of MMP-9 in the chick embryo. Therefore chicken heterophils could be identified by staining with a specific anti-chicken MMP-9 antibody (Zijlstra et al. 2006). On the other hand, inflammatory monocytes are the major source of MMP-13 in the chick embryo and thus, could be easily identified by immunostaining with anti-MMP-13 antibody (Zijlstra et al. 2004). Immunohistochemical analysis of primary CAM tumors developed from HT-1080 variants definitively indicated that HT-hi/diss cells were much more efficient in the induction of heterophil and monocyte/macrophage influxes compared with HT-lo/diss cells (Fig. 7a, b). Quantitation of inflammatory cell density performed in the images of tumor sections confirmed the histological observation by demonstrating a five to ten fold differential in heterophil and monocyte influxes at different portions of primary tumors, including tumor/stroma border (Fig. 7, bottom graphs). 
Fig. 8 Immunohistochemical staining (brown) of blood vessels in HT-lo/diss (a) and HT-hi/ diss (b) tumors developed on the CAM of chick embryos. Paraffin sections were stained with Sambuco negro agglutinin specifically binding to chicken endothelial cells. Counterstaining was performed with Mayer's hematoxylin, $\times 100$. c Quantitation of lumina-containing blood vessels in SNA-stained sections of CAM tumors. d Quantitation of angiogenic blood vessels induced by HT-lo/diss and HT-hi/ diss cells as determined in the collagen onplant assay (Deryugina and Quigley 2008)

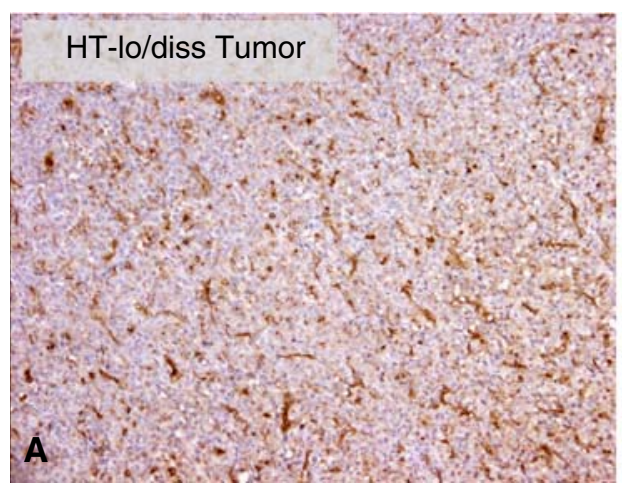

Blood Vessels in CAM Tumors

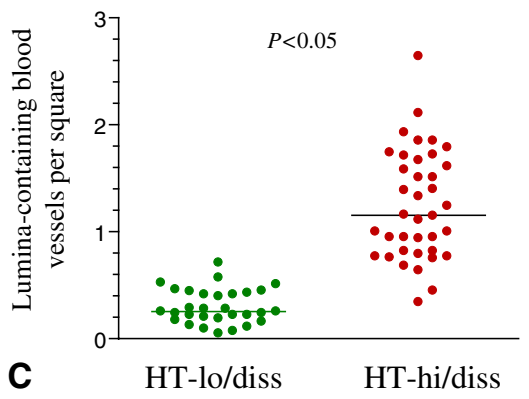

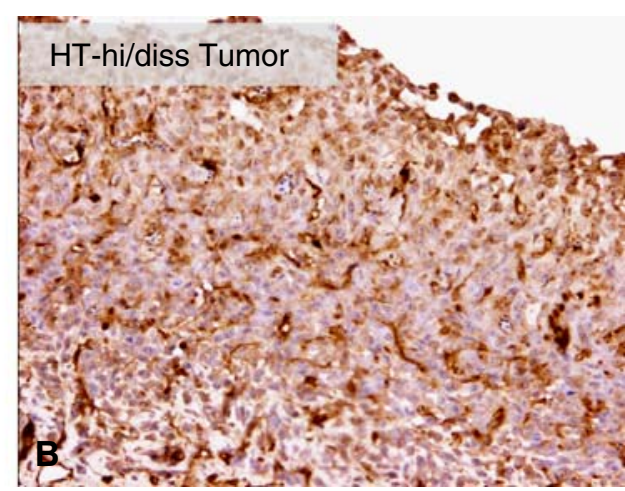

Angiogenic Vessels in CAM Onplants

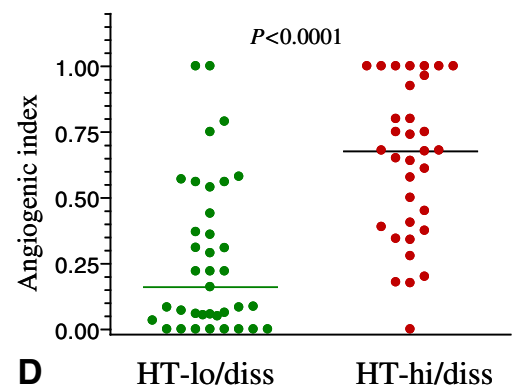

Chicken heterophils and monocytes provide rich sources of highly pro-angiogenic MMPs and growth factors (Harmon 1998; Kogut et al. 2005; Poh et al. 2008); therefore their influxes might facilitate an angiogenic response and result in higher levels of angiogenesis in HT-hi/diss tumors as compared to HT-lo/diss counterparts. Whether this inflammatory influx was associated with higher rate of blood vessel development, was analyzed immunohistochemically in primary tumors developed from HT-1080 dissemination variants. Staining with SNA demonstrated a significant difference in intra-tumoral blood vessel density (Fig. 8a, b). Quantitative analysis has confirmed that HT-hi/diss tumors elicit 2-3 times more lumina-containing blood vessels as compared with HT-lo/diss tumors (Fig. 8c, d). Importantly, this differential in vascular density within CAM tumors is also manifested in an independent angiogenesis assay in which the angiogenic potential of tumor cells is quantitatively assessed in collagen onplants grafted on the CAM (Deryugina and Quigley 2008). In view of the vascular route for tumor cell dissemination in the spontaneous metastasis model, it is appealing to suggest that newly-formed angiogenic intratumoral blood vessels might serve as conduits for intravasation and metastatic dissemination of HT-hi/diss cells.

Circulating human tumor cells in the vasculature of chick embryos during spontaneous metastasis

Although being rare due to rapid vascular arrest, circulating tumor cells can be identified in the peripheral blood of cancer patients especially at the late stages of disease
(Gallagher et al. 2008; Hasselmann et al. 2001; He et al. 2008; Maheswaran et al. 2008). The size of aggressive tumors and time of their development also correlate with the appearance and increasing frequency of tumor cells in the circulation in mice (Allan et al. 2005; Scatton et al. 2006). High levels of intravasation exhibited by HT-hi/diss cells prompted us to attempt detection of circulating human tumor cells during spontaneous metastasis in the chick embryo. This was accomplished by employing a newlydeveloped fiber-optic array scanning technology (FAST), capable of detecting circulating human tumor cells in cancer patients with exceptional sensitivity (Krivacic et al. 2004).

To identify spontaneously intravasated HT-hi/diss cells in circulation, peripheral blood was collected from the embryos bearing developing HT-hi/diss tumors and processed for FAST detection. Peripheral blood cells were distributed on glass slides and immunostained with antihuman CD44 mAb 29-7, specifically binding to human cells and having no cross-reactivity with chicken cells. Kinetic analysis demonstrated that the first circulating HThi/diss cells could be detected as soon as 3 days after cell grafting on the CAM. When peripheral blood was collected from the embryos 4-6 days following grafting, circulating tumor cells were repeatedly detected by a FAST cytometer (Fig. 9). The frequency of circulating HT-hi/diss cells was maximal on day 4 , coinciding with the onset of intravasation as determined in the distal CAM by qPCR (Deryugina et al. 2005).

The unambiguous detection of human cells that actually are present in the host circulation provides strong evidence 
Fig. 9 Detection of intravasated HT-hi/diss cells in circulation. Examples of human tumor cells (red) detected in the peripheral blood of the chick embryo. Peripheral blood (approximately $5 \mathrm{ml}$ ) was collected from allantoic vein 4 days after grafting HT-hi/diss cells $\left(4 \times 10^{5}\right)$ on the CAM of 10-day-old chick embryos. Peripheral blood was separated on discontinuous gradient of Histopaque. The cells from the band containing white blood cells and putative tumor cells were distributed on the slides, immunostained with humanspecific mAb 29-7 against CD44, and processed by a FAST cytometer. Blue cell nuclei stained with DAPI (most stained nuclei represent chicken nucleated erythrocytes), $\times 400$
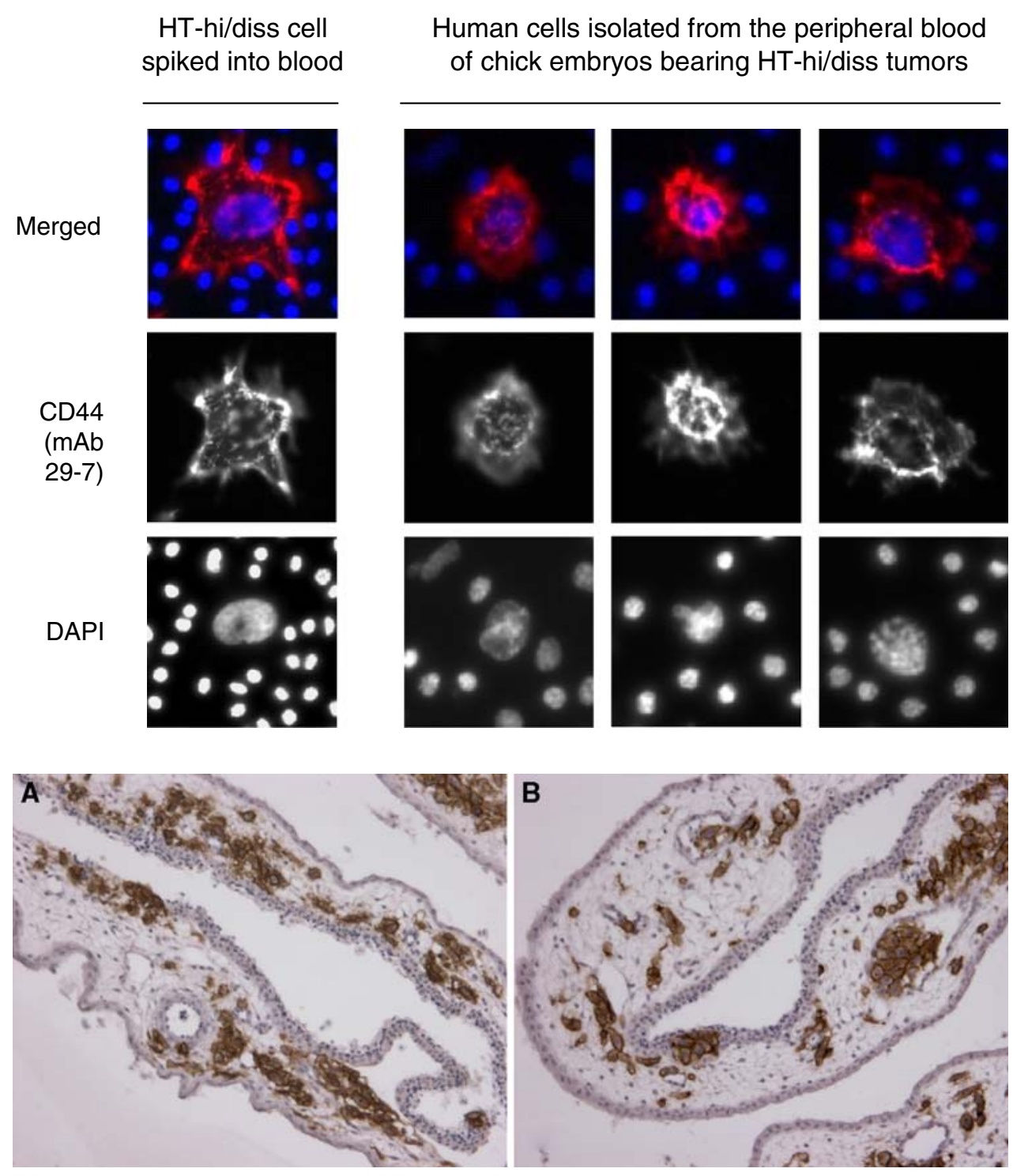

Fig. 10 Dissemination of HT1080 intravasation variants in the experimental metastasis CAM model. Human HT-lo/diss (a) and HT-hi/diss (b) cells were inoculated i.v. into day $12 \mathrm{em}$ bryos and visualized within the CAM tissue 5 days later by immunostaining with mAb 29-7 specific to human CD44 (brown), $\times 200$ that dissemination of tumor cells from the primary CAM tumors indeed involves intravasation, i.e., that HT-hi/diss cells actively enter the vasculature of the chick embryo. It also emphasizes that the spontaneous human tumor/chick embryo model truly recapitulates this clinically established aspect of the metastatic cascade.

Tumor cell extravasation and colonization in the chick embryo experimental metastasis model

To study the events following tumor cell entry into the vasculature, i.e., tumor cell survival in circulation, vascular arrest, extravasation and the ability to form micrometastases, tumor cells are inoculated directly into the circulation, thereby surpassing early steps of spontaneous dissemination. Comparison of tumor cell metastatic behavior in both spontaneous and experimental metastasis models is very informative since it allows one to pin-point the steps of the dissemination cascade where non-metastatic and metastatic cells are functionally different. Thus, the HT-1080 dissemination variants, which exhibit 50-100fold differential in spontaneous metastasis, were remarkably similar in their colonization abilities in the experimental metastasis setting. Immunohistochemical analysis demonstrated that within 5 days after cell inoculation, both HThi/diss and HT-lo/diss cells formed vascular and stromal foci of similar size and distribution within the mesoderm (Fig. 10a, b). Inoculated i.v. cells could be found localized intravascularly or just beneath the ectoderm plexus as extravasated singular cells or further in the mesoderm stroma as multicellular foci. These findings affirmed that HT-1080 dissemination variants were indeed different in their intravasation capacity, i.e., in the ability to successfully perform during early stages of metastasis, after primary tumor formation, but before vascular arrest, extravasation and foci formation. 
Fig. 11 Analysis of experimental metastasis of congenic pair of colon carcinoma cell lines, SW480 and SW620, by AluqPCR (a), live microscopy of green fluorescent-tagged cells (b) and histological examination over time $(\mathbf{d}), \times 100(\mathbf{b})$, and $\times 40(\mathbf{c})$

\section{Experimental Metastasis}
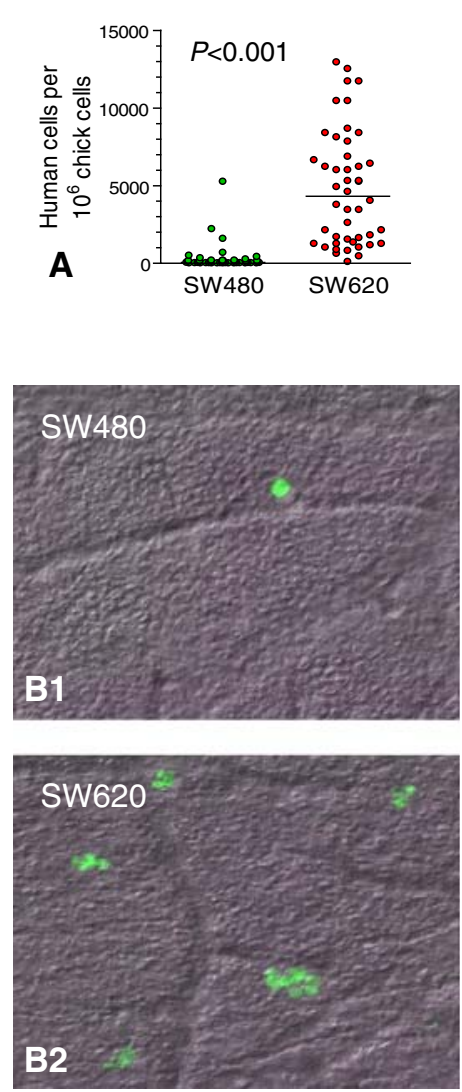

SW480
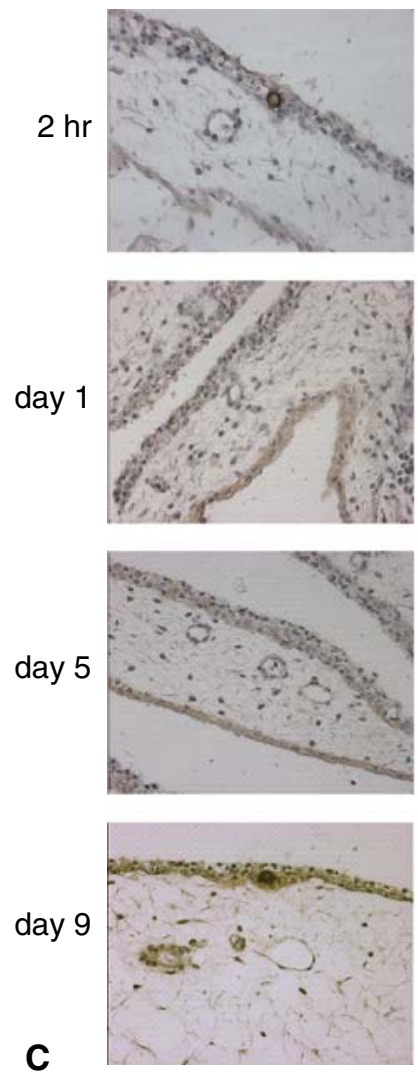

SW620
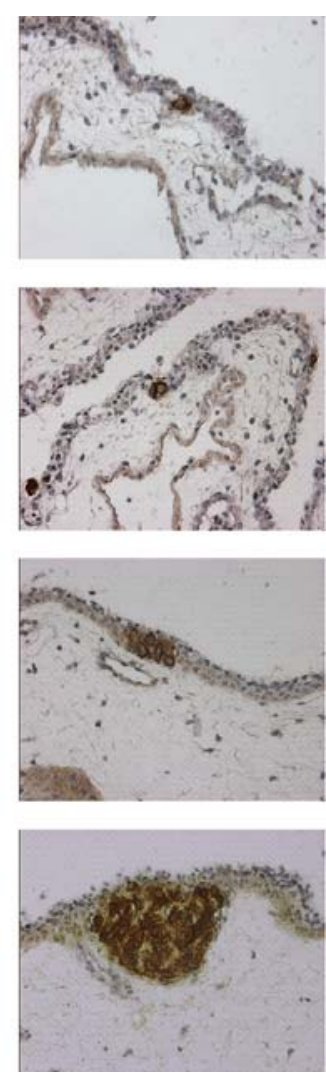

Compelling results could be obtained by detailed histological analysis of congenic tumor cell lines differing significantly in the outcome of experimental metastasis. One such rare pair of cell lines is represented by congenic colon carcinoma cells originating from a primary tumor and recurrent metastasis of the same patient, i.e. SW480 and SW620 cells (Leibovitz et al. 1976). When these two cell lines are injected i.v. into chick embryos, only SW620 cells give rise to high levels of colonization as measured by Alu qPCR (Fig. 11a). To delineate the mechanisms underlying the success of SW620 cells in experimental metastasis and the lack thereof in SW480 cells, we performed detailed kinetic immunohistochemical analysis of tumor cell colonization. Colon carcinoma tumor cells were stained by pan-cytokeratin antibodies and their fate following arrest in the CAM monitored until day 9 after cell inoculation (Fig. 11b). When SW480 and SW620 cells were labeled with the green fluorescent tracker, histological examination of the whole mount CAM preparations by DIC-fluorescent microscopy $48 \mathrm{~h}$ after cell inoculation demonstrated few SW480 cells, while SW620 cells already formed muticellular microfoci (Fig. 11b). Remarkably, SW480 and 620 cells appeared very similar in their initial arrest in the CAM, thus excluding the possibility that SW480 cells were just incapable of survival in circulation. Both cell types were found as singular cells trapped in the ectodermal capillary plexus (Fig. 11c). However, while SW620 cells gradually generated metastatic foci expanding into the CAM mesoderm, SW480 cell were not detected after $48 \mathrm{~h}$ and only occasionally found on day 8-9 after cell injections. These findings suggested that SW480 cells undergo rapid clearance or apoptosis within first $24-72 \mathrm{~h}$ after inoculation in the CAM vasculature.

Close histological evaluation pointed to increased blood vessel density around SW620 micro foci, therefore suggesting that SW620 cells might have higher angiogenic potential as compared with SW480 cells. This suggestion was first confirmed by direct comparison of angiogenic potential of the two cell lines in our collagen onplant angiogenesis assay (Deryugina and Quigley 2008) and then, by inhibiting experimental metastasis of SW620 cells by function-blocking $\mathrm{mAb}$ specifically targeting human VEGF (unpublished data). In view of the known correlation between high VEGF production by metastatic colon carcinomas, including SW620 cells, and high levels of angiogenesis in colon carcinoma metastases, our findings represent a pertinent example of successful use of the chick embryo model system to study specific pathways involved human tumor cell dissemination.

Our recent study of the role of CDCP1 in tumor cell vascular dissemination represents an example of the power 
of histological examination in identifying the physiological mechanism underlying the functionality of metastasisrelated molecules. CDCP1 is a relatively new member of CUB domain-containing proteins, discovered in our laboratory during the course of generating metastasis-blocking antibodies by a process of subtractive immunization (Zijlstra et al. 2003). CDCP1 was identified as an antigen precipitated by a subtractive immunization mAb 41-2 (Hooper et al. 2003). Although it is widely expressed among different normal tissues, including stem cells and cell progenitors of hematopoietic origin, CDCP1 expression has been shown elevated in a number of carcinomas (Perry et al. 2007; Scherl-Mostageer et al. 2001; Uekita et al. 2008). CDCP1 has been implicated in tumor cell functions associated with the capacity of tumor cells to withstand anoikis (Uekita et al. 2007), however, the precise role of CDCP1 in metastasis has not been elucidated in sufficient detail.

We have attempted to identify the step(s) in the metastatic cascade where CDCP1 functions as a pro-metastatic molecule and to mechanistically address the functional role of CDCP1 during experimental metastasis. To achieve these goals, we generated HeLa cells overexpressing CDCP1 (HeLa-CDCP1) and took advantage of our antiCDCP1 function-blocking mAb 41-2. When the embryos were inoculated with HeLa-CDCP1 cells, there was a significant, two- to threefold reduction in the levels of colonization if the recipients were treated with mAb 41-2 as compared to the mouse IgG control. However, extending Alu qPCR data, immunohistochemical analyses of the CAM demonstrated that 41-2-mediated inhibition of colonization was associated with the reduction of total numbers of micro metastatic foci rather than with the decrease in the number of cells comprising individual focus. This finding suggested that the function-blocking anti-CDCP1 mAb 41-2 targets single CDCP1-expressing cells soon after they are arrested in the ectoderm plexus or at early stages of extravasation (unpublished data). To verify this hypothesis, HeLa-CDCP1 cells were pre-labeled with green fluorescent tracker and injected with mAb 41-2 or control IgG, while the vasculature of chick embryos was highlighted with the red fluorescence-tagged lectin (Fig. 12). Kinetic immunofluorescence analysis of HeLaCDCP1 cell distribution within the CAM vasculature indicated rapid vascular arrest of injected cells within $2 \mathrm{~h}$ after inoculation regardless of the antibody specificity. However, there was a significant increase in the percentage of severely fragmented HeLa-CDCP1 cells $12 \mathrm{~h}$ following injection with mAb 41-2. Consistent with our suggestion, HeLa-CDCP1 cells were still singular and appeared as extravasating from the ectodermal capillary plexus. The fragmented cells were presented by apoptotic bodies, suggesting that ligation of cell surface-expressed CDCP1
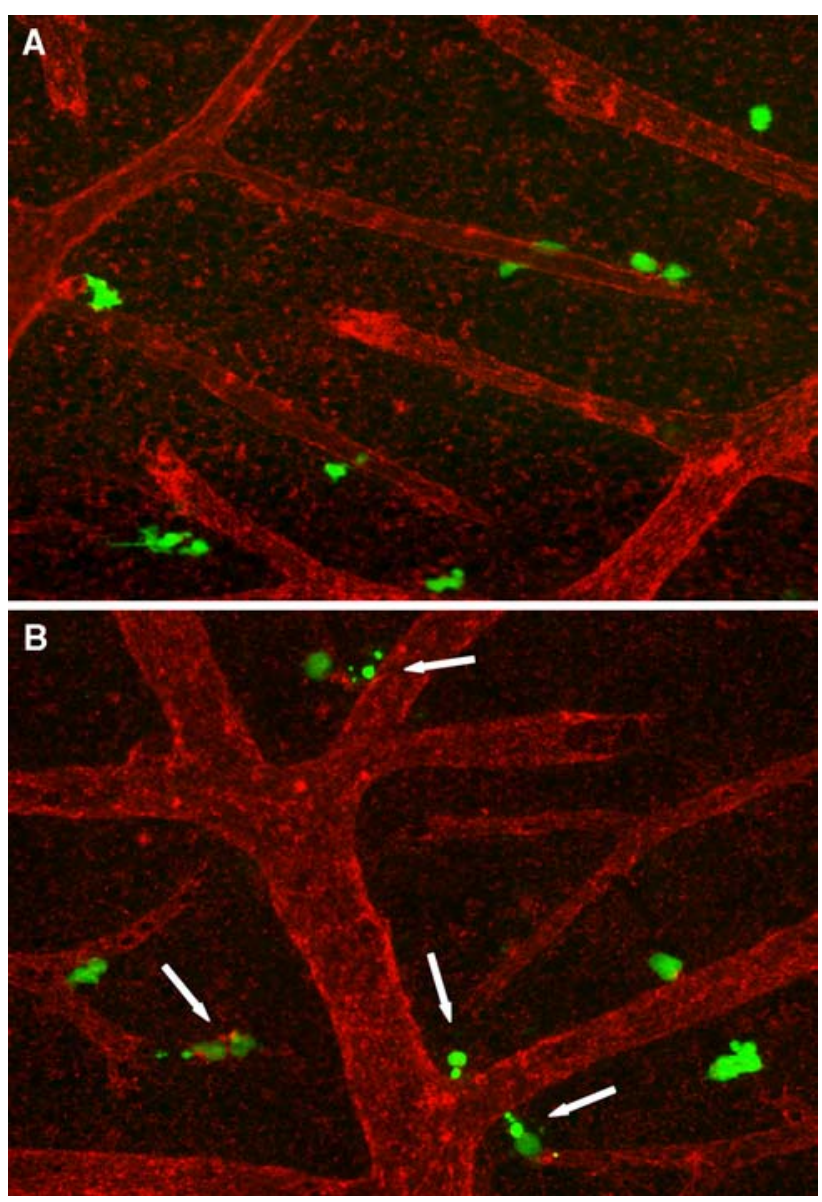

Fig. 12 Induction of HeLa-CDCP1 cell fragmentation by metastasisblocking anti-CDCP1 mAb 41-2. HeLa cells transfected with CDCP1 were labeled with green fluorescent Tracker and inoculated i.v. into chick embryos along with control IgG (a) or anti-CDCP1 mAb 41-2 (b). Twelve hours after cell injections, the embryos were inoculated with red-fluorescent LCA to highlight the CAM vasculature. HeLaCDCP1 cells were visualized in live, non-fixed whole mount preparations of the CAM. Arrows point to fragmented cells apparently undergoing apoptosis due to ligation of CDCP1 with mAb 41-2, $\times 200$

resulted in the increase of apoptosis (Fig. 12). This conclusion, brought about by morphological examination of tumor cell behavior during time course analysis, was later confirmed in selected in vitro settings recapitulating apoptosis-enhancing effects of mAb 41-2 on tumor cells under pro-apoptotic conditions.

Altogether, these findings illustrate that histological analysis effectively complement CAM metastasis model systems and provide insightful views on the functional mechanisms of individual metastasis-related molecules as well as specific behavioral patterns of tumor cells during individual stages of metastatic dissemination.

Acknowledgments The authors would like to acknowledge Dr. Tatyana Kupriyanova, Dr. Nicole Lazarus, Dr. Veronica Ardi and Ms. Juneth Partridge for their expertise in preparation of histological samples and live imaging of cells. 


\section{References}

Allan AL, Vantyghem SA, Tuck AB, Chambers AF, Chin-Yee IH, Keeney M (2005) Detection and quantification of circulating tumor cells in mouse models of human breast cancer using immunomagnetic enrichment and multiparameter flow cytometry. Cytometry A 65:4-14

Armstrong PB, Quigley JP, Sidebottom E (1982) Transepithelial invasion and intramesenchymal infiltration of the chick embryo chorioallantois by tumor cell lines. Cancer Res 42:1826-1837

Balkwill F, Charles KA, Mantovani A (2005) Smoldering and polarized inflammation in the initiation and promotion of malignant disease. Cancer Cell 7:211-217

Condeelis J, Pollard JW (2006) Macrophages: obligate partners for tumor cell migration, invasion, and metastasis. Cell 124:263-266

de Visser KE, Eichten A, Coussens LM (2006) Paradoxical roles of the immune system during cancer development. Nat Rev 6:24-37

Deryugina EI, Quigley JP (2008) Chick embryo chorioallantoic membrane models to quantify angiogenesis induced by inflammatory and tumor cells or purified effector molecules. Methods Enzymol (in press)

Deryugina EI, Zijlstra A, Partridge JJ, Kupriyanova TA, Madsen MA, Papagiannakopoulos T, Quigley JP (2005) Unexpected effect of matrix metalloproteinase down-regulation on vascular intravasation and metastasis of human fibrosarcoma cells selected in vivo for high rates of dissemination. Cancer Res 65:10959-10969

Gallagher DJ, Milowsky MI, Ishill N, Trout A, Boyle MG, Riches J, Fleisher M, Bajorin DF (2008) Detection of circulating tumor cells in patients with urothelial cancer. Ann Oncol. Accessed 3 Oct 2008. doi:10.1093/annonc/mdn627

Harmon BG (1998) Avian heterophils in inflammation and disease resistance. Poult Sci 77:972-977

Hasselmann DO, Rappl G, Rossler M, Ugurel S, Tilgen W, Reinhold U (2001) Detection of tumor-associated circulating mRNA in serum, plasma and blood cells from patients with disseminated malignant melanoma. Oncol Rep 8:115-118

He W, Kularatne SA, Kalli KR, Prendergast FG, Amato RJ, Klee GG, Hartmann LC, Low PS (2008) Quantitation of circulating tumor cells in blood samples from ovarian and prostate cancer patients using tumor-specific fluorescent ligands. Int J Cancer 123:19681973

Hooper JD, Zijlstra A, Aimes RT, Liang H, Claassen GF, Tarin D, Testa JE, Quigley JP (2003) Subtractive immunization using highly metastatic human tumor cells identifies SIMA135/CDCP1, a $135 \mathrm{kDa}$ cell surface phosphorylated glycoprotein antigen. Oncogene 22:1783-1794

Kogut MH, Rothwell L, Kaiser P (2005) IFN-gamma priming of chicken heterophils upregulates the expression of proinflammatory and Th1 cytokine mRNA following receptor-mediated phagocytosis of Salmonella enterica serovar enteritidis. J Interferon Cytokine Res 25:73-81

Krivacic RT, Ladanyi A, Curry DN, Hsieh HB, Kuhn P, Bergsrud DE, Kepros JF, Barbera T, Ho MY, Chen LB, Lerner RA, Bruce RH (2004) A rare-cell detector for cancer. Proc Natl Acad Sci USA 101:10501-10504

Leibovitz A, Stinson JC, McCombs WB 3rd, McCoy CE, Mazur KC, Mabry ND (1976) Classification of human colorectal adenocarcinoma cell lines. Cancer Res 36:4562-4569
Maheswaran S, Sequist LV, Nagrath S, Ulkus L, Brannigan B, Collura CV, Inserra E, Diederichs S, Iafrate AJ, Bell DW, Digumarthy S, Muzikansky A, Irimia D, Settleman J, Tompkins RG, Lynch TJ, Toner M, Haber DA (2008) Detection of mutations in EGFR in circulating lung-cancer cells. N Engl J Med 359:366-377

Melkonian G, Munoz N, Chung J, Tong C, Marr R, Talbot P (2002) Capillary plexus development in the day five to day six chick chorioallantoic membrane is inhibited by cytochalasin D and suramin. J Exp Zool 292:241-254

Murphy JB (1913) Transplantability of tissues to the embryo of foreign species Its bearing on questions of tissue specificity and tumor immunity. J Exp Med 17:482-493

Ossowski L, Reich E (1980) Experimental model for quantitative study of metastasis. Cancer Res 40:2300-2309

Perry SE, Robinson P, Melcher A, Quirke P, Buhring HJ, Cook GP, Blair GE (2007) Expression of the CUB domain containing protein 1 (CDCP1) gene in colorectal tumour cells. FEBS Lett 581:1137-1142

Poh TY, Pease J, Young JR, Bumstead N, Kaiser P (2008) Re-evaluation of chicken CXCR1 determines the true gene structure: CXCLi1 (K60) and CXCLi2 (CAF/interleukin-8) are ligands for this receptor. J Biol Chem 283:16408-16415

Romanoff AL (1960) The avian embryo. The Macmillan Co., New York

Scatton O, Chiappini F, Riou P, Marconi A, Saffroy R, Bralet MP, Azoulay D, Boucheix C, Debuire B, Uzan G, Lemoine A (2006) Fate and characterization of circulating tumor cells in a NOD/ SCID mouse model of human hepatocellular carcinoma. Oncogene 25:4067-4075

Scherl-Mostageer M, Sommergruber W, Abseher R, Hauptmann R, Ambros P, Schweifer N (2001) Identification of a novel gene, CDCP1, overexpressed in human colorectal cancer. Oncogene 20:4402-4408

Schmid CW, Jelinek WR (1982) The Alu family of dispersed repetitive sequences. Science (NY) 216:1065-1070

Uekita T, Jia L, Narisawa-Saito M, Yokota J, Kiyono T, Sakai R (2007) CUB domain-containing protein 1 is a novel regulator of anoikis resistance in lung adenocarcinoma. Mol Cell Biol 27:7649-7660

Uekita T, Tanaka M, Takigahira M, Miyazawa Y, Nakanishi Y, Kanai Y, Yanagihara K, Sakai R (2008) CUB-domain-containing protein 1 regulates peritoneal dissemination of gastric scirrhous carcinoma. The American journal of pathology 172:1729-1739

van Kempen LC, de Visser KE, Coussens LM (2006) Inflammation, proteases and cancer. Eur J Cancer 42:728-734

Zijlstra A, Aimes RT, Zhu D, Regazzoni K, Kupriyanova T, Seandel M, Deryugina EI, Quigley JP (2004) Collagenolysis-dependent angiogenesis mediated by matrix metalloproteinase-13 (collagenase-3). J Biol Chem 279:27633-27645

Zijlstra A, Seandel M, Kupriyanova TA, Partridge JJ, Madsen MA, Hahn-Dantona EA, Quigley JP, Deryugina EI (2006) Proangiogenic role of neutrophil-like inflammatory heterophils during neovascularization induced by growth factors and human tumor cells. Blood 107:317-327

Zijlstra A, Testa JE, Quigley JP (2003) Targeting the proteome/epitome, implementation of subtractive immunization. Biochem Biophys Res Commun 303:733-744 